\title{
Factors associated with immunization of children in Kaduna State, Nigeria, 2016
}

\author{
Lydia A. Taiwo*1, 2, 3, Aisha A. Abubakar², Endie Waziri ${ }^{1,2}$, Lilian A. Okeke ${ }^{1}$ and \\ Suleiman H. Idriss ${ }^{2}$
}

${ }^{1}$ Field Epidemiology, Nigeria Field Epidemiology and Laboratory Training Program, Abuja, Nigeria; ${ }^{2}$ Ahmadu Bello University Zaria, Nigeria, Abuja, Nigeria; ${ }^{3}$ Federal Ministry of Health, Abuja, Nigeria

\section{Objective}

1. To assess the knowledge, perception, and practices of mothers/ caregivers on vaccine preventable diseases in children aged 12-23 months in Kaduna State, Nigeria

2. To determine the immunization coverages in Kaduna State, Nigeria

3. To determine the sources of information on routine immunization among mothers/caregivers of children aged 12-23months in the study area

\section{Introduction}

Immunization is one of the safest and most effective interventions to prevent disease and early child death ${ }^{1}$. Although, about three quarters of the world's child population is reached with the required vaccines, only half of the children in Sub-Saharan Africa get access to basic immunization ${ }^{2}$. A substantial number of children worldwide do not complete immunization schedules because neither health services nor conventional communication mechanisms regularly reach their communities ${ }^{3}$. Separate studies in Australia and Papua New Guinea have shown that knowledge gaps underlie low compliance with vaccination schedules ${ }^{3,4}$. Mothers are less likely to complete immunization schedules if they are poorly Informed about the need for immunization, logistics (which includes time, date, and place of vaccination), and the appropriate series of vaccines to be followed $^{5,6}$. Although knowledge in itself is insufficient to create demand, poor knowledge about the need for vaccination and when the next vaccination is due is a good indicator of poor compliance ${ }^{7}$. Up-to-date, complete, and scientifically valid information about vaccines can help parents to make informed decisions ${ }^{8}$.

Immunity gap created by this low immunization coverage in Northern Nigeria favors the emergence and transmission of some vaccine preventable diseases (VPDs) especially measles and polio?.

\section{Methods}

A cross-sectional descriptive study was conducted using multistage sampling technique; 379 mothers/caregivers with children aged 12-23 months were recruited. Data collection was done using semi structured interviewer-administered questionnaire and analyzed using Epi info ${ }^{\mathrm{TM}}$ version 7. Descriptive statistics using absolute numbers and proportions and Odds ratio/Chi2 were determined between variables and $\mathrm{p} \leq 0.05$ was considered statistically significant. Multivariate analysis was conducted using logistic regression.

\section{Results}

Mean age of respondents was $28.6(\mathrm{SD}= \pm 6.6), 245(64.7 \%)$ practiced Islam, $128(33.8 \%)$ completed Secondary school, $246(64.9 \%)$ unemployed, 361(92.3\%) were married and 186(49.1\%) were from rural settlements. Among the children whose mothers/ caregivers were interviewed, 163(43.01\%) were between aged 16-19 months old while most $238(62.80 \%)$ fell within the birth order of 2 nd $-5^{\text {th }}$ child. Only $59(15.6 \%)$ of these children were found to be fully immunized, evidenced by vaccination card history. Majority of respondents $244(64.4 \%)$ had unsatisfactory knowledge while $197(55.4 \%)$ and 204(54.0\%) exhibited poor perception and bad practices respectively, regarding routine immunization. Commonest source of information was radio 69(61.61\%). Educational status $[\mathrm{OR}=1.9(95 \% \mathrm{CI}: 1.1-3.3)]$ and good perception $[\mathrm{OR}=2.6$ (95\%CI:1.5-4.5)] of mothers were found to be associated with getting information on routine immunization within 12 months prior to this study while Polygamous family setting [OR=0.6(95\%CI:0.2-0.6)], unsatisfactory knowledge $[\mathrm{OR}=0.3(95 \% \mathrm{CI}: 0.2-0.7)]$ and bad practices $[\mathrm{OR}=0.5(95 \% \mathrm{CI}: 0.3-0.9)]$ of mothers were independently associated with lack of information on routine immunization.

\section{Conclusions}

There is low immunization coverage in this community. Mother's educational status, family setting, knowledge, perception and practices about immunization are important factors that influence access to information on routine immunization.

\section{Keywords}

Routine immunization; Information; Mothers; Knowledge

\section{Acknowledgments}

1. Nigerian Field Epidemiology and Laboratory Training Programme

2. State Ministry of Health, Kaduna, Nigeria

\section{References}

1. WHO/IVB. Periodic Intensification of routine immunization: Lessons Learned and Implications for Action 2009; p3-12. www.who.int/ vaccines-documents accessed 16/7/14.

2. Bond L., Nolan T., Pattison P., and Carlin J. Vaccine preventable diseases and immunizations: A qualitative study of mothers' perceptions of severity, susceptibility, benefits and barriers. Australian/New Zealand Journal of Public Health 1998; 22 (4): 441-6.

3. Bukenya, G. B. \& Freeman, P. A. Possible reasons for non-completion of immunization in an urban settlement of Papua New Guinea. Papua New Guinea Medical Journal 1991; 34(1): 22-5.

4. Eng E., Naimoli J., Naimoli G., Parker K.A., and Lowenthal N. The acceptability of childhood immunization to Togolese mothers: A sociobehavioral perspective. Health Education Quarterly 1991; 18 (1): 97-110.

5. Khanom, K. and Salahuddin, A. K. A study of an educational programme on immunization behavior of parents. Bangladesh Medical Research Council Bulletin 1983; 9:18-24.

6. Waisbord, S. \& Larson, H. Why Invest in Communication for Immunization: Evidence and Lessons Learned. A joint publication of the Health Communication Partnership based at Johns Hopkins Bloomberg School of Public Health/Center for Communication Programs (Baltimore) and the United Nations Children's Fund (New York), June, 2005.

7. Greenough, P. Global immunization and culture: Compliance and resistance in large-scale public health campaigns. Social Science \& Medicine 1995; 41 (5): 605-607.

8. Offit P.A. \& Coffin S.E. Communicating science to the public: MMR vaccine and austim. Vaccine 2003; 22(1):1-6.

9. Clements C.J. \& Ratzan S. Misled and confused? Telling the public about MMR vaccine safety; Measles, mumps, and rubella. Journal of Medical Ethics 2003; 29(1):22-6.

\section{*Lydia A. Taiwo \\ E-mail: shallisbabe@yahoo.com}

